\title{
The Efficacy and Safety of Hydroxychloroquine in Patients with COVID-19: A Multicenter National Retrospective Cohort
}

\author{
Abdulkarim Abdulrahman · Islam AlSayed · Marwa AlMadhi • \\ Jumana AlArayed · Sara Jaafar Mohammed · Aesha Khalid Sharif • \\ Khadija Alansari · Abdulla Ismael AlAwadhi · Manaf AlQahtani (D)
}

Received: November 20, 2020 / Accepted: January 6, 2021 / Published online: January 23, 2021

(C) The Author(s) 2021

\section{ABSTRACT}

Introduction: Hydroxychloroquine (HCQ) is an antimalarial drug that received worldwide news and media attention in the treatment of patients with coronavirus disease 2019 (COVID19). This drug was used on the basis of its

Supplementary Information The online version contains supplementary material available at https:// doi.org/10.1007/s40121-021-00397-8.

A. Abdulrahman · A. I. AlAwadhi · M. AlQahtani $(\bowtie)$

National Taskforce for Combating the Coronavirus (COVID-19), Manama, Bahrain

e-mail: mqahtani@rcsi-mub.com

A. Abdulrahman

Mohammed Bin Khalifa Cardiac Centre, Riffa, Bahrain

I. AlSayed · S. J. Mohammed · K. Alansari

King Hamad University Hospital, Busaiteen, Bahrain

M. AlMadhi

School of Medical Sciences, Faculty of Biology,

Medicine and Health, University of Manchester,

Manchester, UK

J. AlArayed - A. K. Sharif

Ministry of Health, Manama, Bahrain

A. I. AlAwadhi · M. AlQahtani

Bahrain Defence Force Hospital, Riffa, Bahrain

M. AlQahtani

Royal College of Surgeons in Ireland - Bahrain, Busaiteen, Bahrain antimicrobial and antiviral properties despite lack of definite evidence of clinical efficacy. In this study, we aim to assess the efficacy and safety of using HCQ in treatment of patients with COVID-19 who were admitted in acute care hospitals in Bahrain.

Methods: We conducted a retrospective cohort study on a random sample of patients admitted with COVID-19 between 24 February and 31 July 2020. The study was conducted in four acute care COVID-19 hospitals in Bahrain. Data was extracted from the medical records. The primary endpoint was the requirement of noninvasive ventilation, intubation, or death. Secondary endpoint was length of hospitalization for survivors. Three methods of analysis were used to control for confounding factors: logistic multivariate regression, propensity score adjusted regression, and matched propensity score analysis.

Results: A random sample of 1571 patients were included, 440 of whom received HCQ (treatment group) and 1131 did not receive it (control group). Our results showed that HCQ did not have a significant effect on primary outcomes due to COVID-19 infection when compared to controls after adjusting for confounders (OR 1.43, 95\% CI 0.85-2.37, $P=0.17$ ). Co-administration of azithromycin had no effect on primary outcomes (OR 2.7, 95\% CI $0.82-8.85, P=0.10)$. HCQ was associated with increased risk of hypoglycemia (OR 10.9, 95\% CI $1.72-69.49, P=0.011$ ) and diarrhea (OR 
2.8, 95\% CI $1.4-5.5, P=0.003)$, but not QT prolongation (OR 1.92, 95\% CI 0.95-3.9, $P=0.06)$ or cardiac arrhythmia (OR 1.06, 95\% CI 0.55-2.05, $P=0.85$ ).

Conclusion: Our results showed no significant beneficial effect of using hydroxychloroquine on the outcome of patients with COVID-19. Moreover, the risk of hypoglycemia due to hydroxychloroquine would possess a significant risk for out-of-hospital use.

Keywords: Azithromycin; COVID-19; Efficacy; Hydroxychloroquine; Respiratory failure; Safety; SARS-CoV-2

\section{Key Summary Points}

Hydroxychloroquine has gained substantial worldwide attention as a possible treatment for COVID-19; however, controversies surrounding its efficacy remain.

Our retrospective cohort study aimed to investigate the effect of hydroxychloroquine use on outcomes in patients with COVID-19.

Hydroxychloroquine did not significantly affect the requirements for ventilatory support or death in patients with COVID19.

Hydroxychloroquine administration was associated with increased risk of developing hypoglycemia and diarrhea.

There was no significant increase in development of QT prolongation and cardiac arrhythmia for patients receiving hydroxychloroquine.

\section{DIGITAL FEATURES}

This article is published with digital features, including a summary slide, to facilitate understanding of the article. To view digital features for this article go to https://doi.org/10.6084/ m9.figshare.13526372.

\section{INTRODUCTION}

An outbreak of severe acute respiratory syndrome coronavirus 2 (SARS-CoV-2), causing the coronavirus disease 2019 (COVID-19), started in December 2019. Almost a year later, we seem to be at the brink of an imminent second wave. Since it was declared a pandemic by the World Health Organization (WHO) in March 2020 [1], it infected more than 52 million people and led to the death of 1.3 million others [2]. With no cure or vaccine identified yet, the health sector moved to repurposing available drugs.

One of the first and most rapidly identified was hydroxychloroquine (HCQ), which was considered owing to its antiviral activity. It was initially developed as an antimalarial drug, and is currently widely used to treat autoimmune diseases like systemic lupus erythematosus and rheumatoid arthritis [3]. The efficacy of HCQ against SARS-CoV-2 was first confirmed in vitro and was reported to mediate its inhibition through the blockage of angiotensin-converting enzyme (ACE) II receptors which facilitate SARS-CoV-2 entry into cells [4]. In addition, HCQ reportedly also disrupted the transport of SARS-CoV-2 from endosomes to endolysosomes, which is necessary for viral release $[4,5]$. HCQ also has immunomodulatory effects such as inhibition of antigen-presenting cell activity, in turn blocking the activation of T cells [6]. This prevents the release of inflammatory cytokines, which causes the "cytokine storm" observed in patients with COVID-19 [6-8]. The US Food and Drug Administration issued an "emergency use authorization" for the use of HCQ for patients with COVID-19, on the basis of these limited results [9], which led to an increase in HCQ use. The first clinical trial studying the use of HCQ to treat COVID-19 was an open-label, non-randomized trial conducted in France. A total of 36 patients received HCQ and 16 controls, with results showing a drop in viral load amongst the HCQ group compared to the controls by day 6 of the trial [10]. Observational studies that followed failed to report a 
therapeutic advantage of the magnitude seen in the French study, instead showing that HCQ has no effect on intubation or mortality amongst patients with COVID-19 [11, 12].

Soon after, studies showing adverse effects of HCQ use started appearing. Concerns regarding safety and efficacy increased after a study was published in the Lancet claiming patients treated with HCQ were at a greater risk of dying at the hospital [13]. A retrospective cohort study of 1438 patients hospitalized in metropolitan New York published in JAMA showed that patients who received HCQ (along with azithromycin) were at increased risk of cardiac arrest [11]. The WHO discontinued the SOLIDARITY trial for HCQ after recommendation from the trial steering committee, on the basis of evidence that HCQ produced little or no reduction in the mortality of hospitalized patients with COVID19 when compared to standard care [14]. Results from the Randomized Evaluation of COVID-19 Therapy (RECOVERY) trial showed that HCQ was not effective in reducing mortality and increasing length of hospital stay [15].

Results from HCQ trials and observational studies have yielded inconsistent results, making the confirmation of its efficacy difficult. This inevitably led to a widespread confusion within the medical community and patients, with some halting its use and others continuing its administration regardless.

An increasing number of studies also reported enhanced HCQ activity when coupled with other drugs. Azithromycin, a macrolide antibiotic commonly used to treat chest infections, was reported to accelerate virus elimination $[10,16,17]$. It was also used in the first HCQ clinical trial on six patients who, by day 6 , tested negative [10]. However, this was a very small sample size, and one of the patients tested positive again on day 8 . The results regarding the combinations of drugs have also been inconsistent and there is no definitive proof of efficacy.

Although HCQ has a better safety clinical profile compared to chloroquine [18], the drug it is derived from, there are many reported risks and side effects of HCQ usage. Along with the common side effects, including nausea and headaches, the most common side effect of
HCQ use is QT interval prolongation and subsequent risk of arrhythmia $[19,20]$. The mechanism by which HCQ initiates arrhythmias is unknown; however, its electrophysiological effects include blocking several currents-funny current, L-type calcium current, and rectifier potassium currents [21]. These lead to sinus bradycardia and repolarization abnormalities, the latter leading to the observed QT prolongation [20]. A clinical trial studying the effects of different chloroquine doses involving 81 patients with COVID-19 in Brazil was prematurely stopped after patients receiving the higher dose $(600 \mathrm{mg}$, twice daily) developed arrhythmia within 2-3 days of starting the trial [22]. Although chloroquine is known to be more toxic than HCQ, the study suggested that both drugs have also been associated with liver and renal impairment [8], both of which have also been reported in patients with COVID-19 [23]. With suggestions that incidence of hepatic malfunctioning increases with COVID-19 infection [24], this side effect of HCQ use could be detrimental. This, and the lack of conclusive evidence for the efficacy of HCQ in treating COVID-19, creates a reluctance amongst the public and the healthcare sector to using it. This is a retrospective observational study that aims to investigate HCQ efficacy on clinical and safety outcomes amongst patients with COVID19.

\section{METHODS}

\section{Study Design and Setting}

A retrospective cohort study was done on patients with COVID-19 in Bahrain. Cases that were admitted to Ministry of Health COVID-19 treatment facilities were included. The four hospitals included were Ebrahim bin Khalil Kanoo COVID-19 Centre, SMC 6th floor COVID-19 Centre, Hereditary Blood Disorder Centre (HBDC) COVID-19 Centre, and Jidhafs COVID-19 Centre. All cases who were admitted to these facilities were confirmed to be infected by SARS-CoV-2 by a polymerase chain reaction (PCR) test of a nasopharyngeal sample. Cases 
admitted between 24 February and 31 July were included.

A random sample of cases who received HCQ and a random sample of cases who did not receive HCQ within the study time period were included. Patients who were started on non-invasive ventilation (NIV), intubated, died, or were transferred to a different facility within $24 \mathrm{~h}$ from admission were excluded from the analysis.

\section{Hydroxychloroquine Exposure}

Labeling patients as "receiving HCQ" depended on whether they received the drug at our study baseline-defined as within $72 \mathrm{~h}$ of admission.

The national Bahrain treatment protocol, developed by the national task force medical team, was issued to all COVID-19 facilities as guidance to healthcare workers for the management of COVID-19. HCQ was suggested for patients with COVID-19 as a therapeutic option. The suggested HCQ regimen was a loading dose of $600 \mathrm{mg}$ twice on day 1 , followed by $400 \mathrm{mg}$ daily for four additional days. Azithromycin at a dose of $500 \mathrm{mg}$ on day 1 and then $250 \mathrm{mg}$ daily for four more days in combination with HCQ was an additional suggested therapeutic option. However, the suggestion of HCQ and/or azithromycin was removed in April after several manuscripts showed lack of benefit from HCQ and a potential risk. Prescribing either or both medications was a decision left to the judgment of the treating team and based on individualization of the patient care.

\section{Data Sources and Variables Assessed}

We obtained data from the I-SEHA electronic medical records. The I-SEHA is a doctor station which provides access to patient records and has all the clinical details of the hospital stay as text files. Data was manually extracted from the electronic records. Five physicians who were assisted by 10 senior medical students reviewed all the cases and filled in an electronic form developed to collect data for this study. The data gathered included patients' demographic details, vital signs, laboratory test results, medication lists, past medical history, clinical severity scale (see the table in the supplementary material), oxygenation requirement on admission, the ratio of the oxygen saturation to the fraction of inspired oxygen $(\mathrm{SpO} 2 / \mathrm{FiO} 2)$ at admission, requirement of ICU care, ventilator use, and outcomes. A complete list of variables collected is available in the supplementary material.

\section{Outcomes}

Primary outcome

The requirement of noninvasive ventilation, intubation, or death. When a patient died after ventilator requirement, the timing of the primary endpoint was defined as the time of the first use of ventilator.

Safety outcomes The development of any of the adverse events during hospital stay, after the prescription of medications. Adverse event included cardiac arrythmia, QT prolongation (> $500 \mathrm{~ms}$ ), diarrhea, and hypoglycemia (defined as glucose levels less than 3.9 $\mathrm{mmol} / \mathrm{L}) 3.6 \mathrm{mmol} / \mathrm{L}$ ).

Secondary outcome The length of stay in days for survivors.

These outcomes will be studied in two contexts: how they compare between control group and HCQ-receiving group; and amongst the HCQreceiving group, how they compare between those also receiving azithromycin and those not receiving it.

\section{Statistical Analysis}

The distribution of treatment groups was summarized. Bivariate associations between the treatment group and the measured patient characteristics were analyzed using chi-squared $\left(\chi^{2}\right)$ tests for categorical variables and $t$ test for 
continuous variables. We also assessed endpoints and adverse events and their associations with the treatment group.

A logistic regression model was used to estimate the relationship between HCQ use and the composite endpoint. A primary multivariable logistic regression model involved demographic factors, clinical factors, and medications.

Propensity score methods were used as well to reduce the effects of confounding and to account for the non-randomized treatment administration of HCQ. The individual propensities for receipt of HCQ treatment were estimated with the use of a multivariable logistic regression model that included pre-treatment variables and predictors and risk for the outcome. Variables used were demographic factors, clinical factors, and chronic diseases status.

An estimation of the association between HCQ use and the primary outcome was assessed by multivariable logistic regression models and the use of two propensity score methods: propensity score matching and the use of the propensity score as an additional covariate in the multivariate logistic regression model for the outcome.

Effect modification was examined for the primary outcome for two variables: (1) HCQ and the baseline severity of disease (whether or not the patient was hypoxic), and (2) the coprescription of azithromycin.

Estimations of the safety and secondary outcome were conducted through the use of the primary analysis, using multivariate regression models.

The STATA software, version 15.1 (StataCorp. 2017. Stata Statistical Software: Release 15. College Station, TX: StataCorp LLC) was used to execute the statistical analyses.

\section{Compliance with Ethics Guidelines}

The protocol and manuscript for this study were reviewed and approved by the National COVID19 Research Committee in Bahrain (Approval Code CRT-COVID2020-061). All methods and retrospective analysis of data were approved by the National COVID-19 Research and Ethics
Committee, and carried out in accordance with the local guideline and ethical guidelines of the Declaration of Helsinki 1975.

\section{RESULTS}

\section{Sample Characteristics}

A total of 1849 cases were reviewed. Of those, 278 were excluded; 57 because of duplication, 79 age less than 18 years, and 34 insufficient information. A further 56 patients were excluded because an endpoint (of ventilatory support or death) was achieved within 1 day, 7 were excluded because of transfer/discharge within 1 day, and 45 were excluded as they received HCQ out of study baseline. Therefore, 1571 cases were included in the study.

Out of the 1571 patients affected with COVID-19 selected in this study, 440 patients received HCQ and 1131 patients did not.

Among the patients who received HCQ. The median time to start HCQ was 1 day from admission (IQR 0-2).

Patients' baseline characteristics (demographic and clinical) according to HCQ exposure are shown in Tables 1 and 2. A propensity matched analysis was conducted to balance the two groups and their characteristics are also shown in Tables 1 and 2.

In the unmatched sample, patients who received HCQ had a significantly higher mean age (43.4 years), were more likely to be Bahraini, and had more comorbidities. Diabetes and hypertension were more common in patients receiving $\mathrm{HCQ}$. The HCQ-receiving patients were more likely to be symptomatic $(68.9 \%$ compared to $61.8 \%$ ). Symptoms of fever, cough, body ache, nausea, and vomiting were more predominant in patients who received HCQ. The HCQ-receiving patients were also more severely ill on admission, as $12.3 \%$ received supplemental oxygen on admission (through nasal cannula, face mask, and nonrebreather mask). 
Table 1 Unmatched and matched patient characteristics

\begin{tabular}{|c|c|c|c|c|c|c|}
\hline \multirow[b]{2}{*}{ Factor } & \multicolumn{3}{|l|}{ Unmatched } & \multicolumn{3}{|l|}{ Matched } \\
\hline & Control & HCQ & $p$-value & Control & HCQ & $p$-value \\
\hline Number & 1131 & 440 & & 223 & 223 & \\
\hline Age:, mean (SD) & $44.6(15.0)$ & $53.4(14.1)$ & $<0.001$ & $52.7(14.4)$ & $50.5(13.8)$ & 0.096 \\
\hline male & $688(60.8 \%)$ & $245(55.7 \%)$ & 0.062 & $131(58.7 \%)$ & $122(54.7 \%)$ & 0.39 \\
\hline Bahraini & $579(51.2 \%)$ & $290(65.9 \%)$ & $<0.001$ & $107(48.0 \%)$ & $152(68.2 \%)$ & $<0.001$ \\
\hline \multicolumn{7}{|l|}{ Number of comorbidities } \\
\hline 0 & $100(8.8 \%)$ & $40(9.1 \%)$ & \multirow[t]{5}{*}{$<0.001$} & $17(7.6 \%)$ & $25(11.2 \%)$ & \multirow[t]{5}{*}{0.008} \\
\hline 1 & $41(3.6 \%)$ & $43(9.8 \%)$ & & $10(4.5 \%)$ & $25(11.2 \%)$ & \\
\hline 2 & $17(1.5 \%)$ & $27(6.1 \%)$ & & $7(3.1 \%)$ & $15(6.7 \%)$ & \\
\hline 3 & $522(46.2 \%)$ & $135(30.7 \%)$ & & $83(37.2 \%)$ & $66(29.6 \%)$ & \\
\hline $4+$ & $451(39.9 \%)$ & $195(44.3 \%)$ & & $106(47.5 \%)$ & $92(41.3 \%)$ & \\
\hline Sickle cell disease & $26(2.3 \%)$ & $6(1.4 \%)$ & 0.24 & $2(0.9 \%)$ & $5(2.2 \%)$ & 0.25 \\
\hline G6PD deficiency & $134(11.8 \%)$ & $29(6.6 \%)$ & 0.002 & $18(8.1 \%)$ & $22(9.9 \%)$ & 0.51 \\
\hline Diabetes mellitus & $273(24.1 \%)$ & $174(39.5 \%)$ & $<0.001$ & $72(32.3 \%)$ & $74(33.2 \%)$ & 0.84 \\
\hline Cardiovascular disease (CVD) & $105(9.3 \%)$ & $53(12.0 \%)$ & 0.10 & $23(10.3 \%)$ & $27(12.1 \%)$ & 0.55 \\
\hline Hypertension & $285(25.2 \%)$ & $176(40.0 \%)$ & $<0.001$ & $79(35.4 \%)$ & $78(35.0 \%)$ & 0.92 \\
\hline Asthma & $42(3.7 \%)$ & $23(5.2 \%)$ & 0.18 & $9(4.0 \%)$ & $12(5.4 \%)$ & 0.50 \\
\hline $\begin{array}{l}\text { Chronic obstructive pulmonary disease } \\
\text { (COPD) }\end{array}$ & $2(0.2 \%)$ & $4(0.9 \%)$ & 0.035 & 0 & 0 & \\
\hline Obesity $(\mathrm{BMI}>=30)$ & $33(2.9 \%)$ & $20(4.5 \%)$ & 0.11 & $7(3.1 \%)$ & $13(5.8 \%)$ & 0.17 \\
\hline Chronic kidney disease (CKD) & $37(3.3 \%)$ & $22(5.0 \%)$ & 0.11 & $12(5.4 \%)$ & $13(5.8 \%)$ & 0.84 \\
\hline $\begin{array}{l}\text { Other chronic lung disease (not asthma } \\
\text { nor COPD) }\end{array}$ & $4(0.4 \%)$ & $2(0.5 \%)$ & 0.77 & $2(0.9 \%)$ & $1(0.4 \%)$ & 0.56 \\
\hline \multicolumn{7}{|l|}{ Smoker } \\
\hline Current & $18(1.9 \%)$ & $11(2.6 \%)$ & 0.52 & $3(1.6 \%)$ & $7(3.3 \%)$ & 0.30 \\
\hline Exsmoker & $14(1.5 \%)$ & $4(0.9 \%)$ & & $3(1.6 \%)$ & $1(0.5 \%)$ & \\
\hline Never & $921(96.6 \%)$ & $414(96.5 \%)$ & & $182(96.8 \%)$ & $207(96.3 \%)$ & \\
\hline
\end{tabular}

G6PD glucose-6-phosphate 1-dehydrogenase, $C V D$ cardiovascular disease, COPD chronic obstructive pulmonary disease, $B M I$ body mass index, $C K D$ chronic kidney disease 


\section{Propensity Score}

The distribution of the estimated propensity scores for receiving HCQ among patients who did and did not receive HCQ is shown in the supplementary material. The $C$-statistic of the propensity score model was 0.83 . In the matched analytic sample, 223 patients were exposed to HCQ and 223 were not exposed. The differences between HCQ and pretreatment variables were attenuated in the propensity score matched samples as compared with the unmatched samples.

\section{Primary Outcome}

During the period of their admission, patients who received HCQ were more likely to develop the composite outcome. Of 440 patients receiving HCQ, 24 (5.45\%) developed the primary outcome of requiring ventilatory support (invasive and non-invasive) or death in comparison to 44 of 1131 patients (3.89\%) who were not treated with HCQ. Table 3 summarizes outcomes in each treatment group.

The difference between the two groups was not significant across the different methods used to control confounders. The primary analysis using multivariate model adjusting for confounding variables showed an odds ratio of 1.43 with a $95 \%$ CI $0.85-2.37, P=0.17$. Other methods of confounding adjustment showed similar and non-significant results. Table 4 summarizes the analysis results.

There was a significant effect modification in patients with COVID-19 receiving HCQ and requiring supplemental oxygen on admission. Significant effect modification was also noted in cases exposed to azithromycin. The supplementary material shows a detailed effect modification analysis.

A total of 179 patients required supplemental oxygen on admission (nasal cannula, face mask, or nonrebreather face mask), of whom 75 received HCQ and 104 did not. Patients who were treated by HCQ were less likely to develop the outcome if they required oxygen on baseline. Fifteen patients in the HCQ group developed the primary outcome (20\%), compared to
30 in the control group (28.85\%). The difference was non-significant in the primary analysis (OR 1.09, 95\% CI 0.38-3.07).

A total of 1392 patients were admitted on room air and did not require supplemental oxygen. Of those, 365 received HCQ and 1027 did not. Nine patient who received HCQ developed the primary outcome $(2.47 \%)$, while 14 patients in the control group developed the outcome $(1.36 \%)$. Treatment with HCQ showed a non-significant increase in odds ratio to develop the primary outcome (OR $2.79,95 \%$ CI 0.92-8.43). Table 5 summarizes the results stratified by oxygen requirement at baseline.

The analysis showed insignificant results when stratified by azithromycin exposure. It was noted that patients who received HCQ and azithromycin had an odds ratio of 2.7 to develop the primary outcome (95\% CI 0.82-8.85). Patients who were treated with HCQ and did not receive azithromycin had an odds ratio of 1.3 to develop the primary outcome (95\% CI 0.44-3.75). Results are summarized in Table 6.

\section{Safety Outcome}

Patients who received HCQ had significantly increased odds ratio to develop hypoglycemia (OR 10.9, 95\% CI 1.72-69.49, $P=0.011$ ) and diarrhea (OR 2.8, 95\% CI 1.4-5.5, $P=0.003$ ). Patients treated with HCQ were more likely to develop QT prolongation (OR 1.92, 95\% CI $0.95-3.9, P=0.06)$ and cardiac arrhythmias (OR 1.06, 95\% CI $0.55-2.05, P=0.85)$; however, these findings were non-significant. Table 7 summarizes the safety endpoints.

\section{Secondary Outcome: Length of Stay in Survived Cases}

The mean length of stay of discharged patients in the study cohort was 10.0 days ( \pm 5.54 days). The minimum stay was 2 days, and the maximum was 57. Patients who received HCQ had a mean stay of 11.3 days (5.65 days) while patients in the control group had a mean stay of 9.5 days (5.41 days). The difference was statistically significant in a two-sided $t$ test $(p<0.001)$. 
Table 2 Unmatched and matched patient clinical characteristics

\begin{tabular}{|c|c|c|c|c|c|c|}
\hline \multirow[t]{2}{*}{ Factor } & \multicolumn{3}{|l|}{ Unmatched } & \multicolumn{3}{|l|}{ Matched } \\
\hline & Control & HCQ & p-value & Control & HCQ & p-value \\
\hline Number & 1131 & 440 & & 223 & 223 & \\
\hline Symptoms on admission: & $699(61.8 \%)$ & $303(68.9 \%)$ & 0.009 & $154(69.1 \%)$ & $151(67.7 \%)$ & 0.76 \\
\hline Fever $(>38 \mathrm{C})$ & $230(20.3 \%)$ & $118(26.8 \%)$ & 0.005 & $48(21.5 \%)$ & $49(22.0 \%)$ & 0.91 \\
\hline Cough & $450(39.8 \%)$ & $218(49.5 \%)$ & $<0.001$ & $108(48.4 \%)$ & $107(48.0 \%)$ & 0.92 \\
\hline Chest pain & $91(8.0 \%)$ & $49(11.1 \%)$ & 0.054 & $23(10.3 \%)$ & $26(11.7 \%)$ & 0.65 \\
\hline Shortness of breath & $210(18.6 \%)$ & $85(19.3 \%)$ & 0.73 & $53(23.8 \%)$ & $48(21.5 \%)$ & 0.57 \\
\hline Loss of smell & $30(2.7 \%)$ & $18(4.1 \%)$ & 0.14 & $4(1.8 \%)$ & $14(6.3 \%)$ & 0.016 \\
\hline Loss of taste & $30(2.7 \%)$ & $17(3.9 \%)$ & 0.21 & $6(2.7 \%)$ & $14(6.3 \%)$ & 0.067 \\
\hline Diarrhea & $61(5.4 \%)$ & $30(6.8 \%)$ & 0.28 & $14(6.3 \%)$ & $16(7.2 \%)$ & 0.71 \\
\hline Nausea or vomiting & $46(4.1 \%)$ & $34(7.7 \%)$ & 0.003 & $9(4.0 \%)$ & $17(7.6 \%)$ & 0.11 \\
\hline Body pain & $167(14.8 \%)$ & $89(20.2 \%)$ & 0.008 & $37(16.6 \%)$ & $49(22.0 \%)$ & 0.15 \\
\hline $\begin{array}{l}\text { Heart rate on admission: bpm, } \\
\text { mean (SD) }\end{array}$ & $85.8(13.7)$ & $86.4(13.6)$ & 0.44 & $85.4(14.3)$ & $86.6(13.0)$ & 0.35 \\
\hline $\begin{array}{l}\text { SBP on admission: } \mathrm{mmHg} \text {, mean } \\
\text { (SD) }\end{array}$ & $129.6(18.4)$ & $133.7(18.3)$ & $<0.001$ & $132.4(20.7)$ & $133.6(18.8)$ & 0.50 \\
\hline $\begin{array}{l}\text { DBP on admission: } \mathrm{mmHg} \text {, } \\
\text { mean }(\mathrm{SD})\end{array}$ & $77.9(11.5)$ & $76.8(11.3)$ & 0.080 & $78.7(10.9)$ & $77.5(11.2)$ & 0.25 \\
\hline $\begin{array}{l}\text { Requirment of oxygen support } \\
\text { on admission }\end{array}$ & $104(9.2 \%)$ & $54(12.3 \%)$ & 0.069 & $28(12.6 \%)$ & $27(12.1 \%)$ & 0.89 \\
\hline \multicolumn{7}{|l|}{ Nasal canula } \\
\hline $\begin{array}{l}\text { Oxygenation device on } \\
\text { admission }\end{array}$ & $43(41.0 \%)$ & $36(66.7 \%)$ & 0.008 & $8(29 \%)$ & $17(63 \%)$ & 0.032 \\
\hline Face mask & $45(42.9 \%)$ & $14(25.9 \%)$ & & $16(57 \%)$ & $7(26 \%)$ & \\
\hline Nonrebreather face mask & $17(16.2 \%)$ & $4(7.4 \%)$ & & $4(14 \%)$ & $3(11 \%)$ & \\
\hline SpO2:FiO2 ratio, mean (SD) & $445.08(71.63)$ & $439.82(71.32)$ & 0.19 & $436.17(82.08)$ & $438.35(75.45)$ & 0.77 \\
\hline $\begin{array}{l}\text { Presence of an elevated } \\
\qquad \mathrm{ALT}>40 \mathrm{U} / \mathrm{L} \text { on admission }\end{array}$ & $249(23.6 \%)$ & $114(26.8 \%)$ & 0.19 & $49(23.2 \%)$ & $70(32.0 \%)$ & 0.043 \\
\hline $\begin{array}{l}\text { Presence of an elevated } \\
\text { creatinine on admission }\end{array}$ & $78(6.9 \%)$ & $52(11.8 \%)$ & 0.001 & $23(10.3 \%)$ & $25(11.2 \%)$ & 0.76 \\
\hline \multicolumn{7}{|l|}{ Chest Xray findings on admission } \\
\hline Pneumonia & $291(31.0 \%)$ & $167(39.5 \%)$ & 0.002 & $59(31.4 \%)$ & $73(33.5 \%)$ & 0.65 \\
\hline Normal & $648(69.0 \%)$ & $256(60.5 \%)$ & & $129(68.6 \%)$ & $145(66.5 \%)$ & \\
\hline
\end{tabular}


Table 2 continued

\begin{tabular}{|c|c|c|c|c|c|c|}
\hline \multirow[t]{2}{*}{ Factor } & \multicolumn{3}{|l|}{ Unmatched } & \multicolumn{3}{|l|}{ Matched } \\
\hline & Control & HCQ & p-value & Control & HCQ & p-value \\
\hline $\begin{array}{l}\text { Hypotension }(\mathrm{SBP}<90 \mathrm{mmHg} \\
\text { or } \mathrm{DBP}<60 \mathrm{mmHg}) \text { on } \\
\text { admission }\end{array}$ & $43(3.8 \%)$ & $14(3.2 \%)$ & 0.56 & $7(3.1 \%)$ & $7(3.1 \%)$ & 1.00 \\
\hline $\begin{array}{l}\text { Tachypnea }(\mathrm{RR}>22) \text { on } \\
\text { admission }\end{array}$ & $20(1.8 \%)$ & $11(2.5 \%)$ & 0.35 & $5(2.2 \%)$ & $8(3.6 \%)$ & 0.40 \\
\hline \multicolumn{7}{|l|}{ Baseline clinical severity scale } \\
\hline Isolated and asymptomatic & $5(0.4 \%)$ & $1(0.2 \%)$ & $<0.001$ & $0(0.0 \%)$ & $1(0.4 \%)$ & 0.33 \\
\hline Isolated mild symptomatic & $4(0.4 \%)$ & $1(0.2 \%)$ & & $2(0.9 \%)$ & $1(0.4 \%)$ & \\
\hline Admitted on room air & $1018(90.0 \%)$ & $363(82.5 \%)$ & & $193(86.5 \%)$ & $182(81.6 \%)$ & \\
\hline Admitted and on oxygen support & $104(9.2 \%)$ & $75(17.0 \%)$ & & $28(12.6 \%)$ & $39(17.5 \%)$ & \\
\hline Admitted and on NIV/HFNC & 0 & 0 & & 0 & 0 & \\
\hline $\begin{array}{l}\text { Admitted and on mechanical } \\
\text { ventilation/ECMO }\end{array}$ & 0 & 0 & & 0 & 0 & \\
\hline $\begin{array}{l}\text { Azithromycin during hospital } \\
\text { stay: }\end{array}$ & $150(13.3 \%)$ & $236(53.6 \%)$ & $<0.001$ & $59(26.5 \%)$ & $64(28.7 \%)$ & 0.60 \\
\hline Kaletra during the hospital stay & $186(16.4 \%)$ & $82(18.6 \%)$ & 0.30 & $38(17.0 \%)$ & $39(17.5 \%)$ & 0.90 \\
\hline Ribavirin during the hospital stay & $180(15.9 \%)$ & $45(10.2 \%)$ & 0.004 & $33(14.8 \%)$ & $29(13.0 \%)$ & 0.58 \\
\hline Steroids during hospital stay & $98(8.7 \%)$ & $66(15.0 \%)$ & $<0.001$ & $19(8.5 \%)$ & $39(17.5 \%)$ & 0.005 \\
\hline Tocilizumab during hospital stay & $31(2.7 \%)$ & $29(6.6 \%)$ & $<0.001$ & $6(2.7 \%)$ & $15(6.7 \%)$ & 0.044 \\
\hline $\begin{array}{l}\text { Received convalescent plasma } \\
\text { transfusion during hospital } \\
\text { stay }\end{array}$ & $19(1.7 \%)$ & $33(7.5 \%)$ & $<0.001$ & $3(1.3 \%)$ & $17(7.6 \%)$ & 0.001 \\
\hline
\end{tabular}

$S B P$ systolic blood pressure, $D B P$ diastolic blood pressure, $S \mathrm{PO} / \mathrm{FiO2}$ ratio of the oxygen saturation to the fraction of inspired oxygen, $A L T$ alanine aminotransferase, $R R$ respiratory rate, $N I V$ non-invasive ventilation, $H F N C$ high-flow nasal cannula oxygenation, ECMO extracorporeal membrane oxygenation

After adjustment for confounders using a multivariate model, HCQ had a higher length of stay by 0.63 days; however, this difference was non-significant ( $95 \% \mathrm{CI}-0.02$ to 1.29$)$. Table 8 summarizes these findings.

Regression models results and details are in the supplementary material.

\section{DISCUSSION}

The results of this study show that, for our studied sample and models used, HCQ did not have a significant effect on primary outcomes (requirement for ventilation or death) due to COVID-19 infection.

Analysis of the demographics of our studied sample showed that patients who received HCQ were significantly of older age, which could be 
Table 3 Outcomes within the unmatched and matched samples

\begin{tabular}{|c|c|c|c|c|c|c|}
\hline \multirow[t]{2}{*}{ Factor } & \multicolumn{3}{|c|}{ Unmatched } & \multicolumn{3}{|l|}{ Matched } \\
\hline & Control & HCQ & $P$ value & Control & HCQ & $P$ value \\
\hline Number & 1131 & 440 & & 223 & 223 & \\
\hline Primary outcome: ventilation or death & $\begin{array}{l}44 \\
\quad(3.9 \%)\end{array}$ & $\begin{array}{l}24 \\
\quad(5.5 \%)\end{array}$ & 0.17 & $7(3.1 \%)$ & $12(5.4 \%)$ & 0.24 \\
\hline Ventilation & $\begin{array}{l}41 \\
(3.6 \%)\end{array}$ & $\begin{array}{l}24 \\
(5.5 \%)\end{array}$ & 0.10 & $7(3.1 \%)$ & $12(5.4 \%)$ & 0.24 \\
\hline Invasive ventilation & $\begin{array}{l}18 \\
(1.6 \%)\end{array}$ & $9(2.0 \%)$ & 0.53 & $3(1.3 \%)$ & $6(2.7 \%)$ & 0.31 \\
\hline Death & $\begin{array}{l}26 \\
(2.3 \%)\end{array}$ & $8(1.8 \%)$ & 0.56 & $6(2.7 \%)$ & $5(2.24 \%)$ & 0.76 \\
\hline Cardiac arrythmia & $\begin{array}{l}38 \\
(3.4 \%)\end{array}$ & 19 & 0.36 & ${ }^{10}(4.5 \%)$ & $9(4.0 \%)$ & 0.81 \\
\hline QT prolongation $>500 \mathrm{~ms}$ & $\begin{array}{l}33 \\
(2.9 \%)\end{array}$ & 22 & 0.044 & $5(2.2 \%)$ & $13(5.8 \%)$ & 0.054 \\
\hline \multicolumn{7}{|l|}{ Adverse events } \\
\hline $\begin{array}{l}\text { Hypoglycemia [blood glucose }<3.9 \mathrm{mmol} / \mathrm{L} \\
(70 \mathrm{mg} / \mathrm{dL})]\end{array}$ & $2(0.2 \%)$ & $7(1.6 \%)$ & $<0.001$ & $1(0.4 \%)$ & $4(1.8 \%)$ & 0.18 \\
\hline Diarrhea & $\begin{array}{l}23 \\
(2.0 \%)\end{array}$ & $\begin{array}{l}27 \\
(6.1 \%)\end{array}$ & $<0.001$ & $5(2.2 \%)$ & $14(6.3 \%)$ & 0.035 \\
\hline
\end{tabular}

associated with a more severe HCQ-requiring presentation. Although this is an extrapolation from our data, age has reportedly been associated with a more severe progression of the disease [25-28]. However, a recent study quantifying the isolated effect of age on severity of COVID-19 outcomes concluded a minimal influence of age after adjusting for important age-dependent risk factors (e.g., diabetes, hypertension, cardiovascular disease (CVD) etc.) [29]. Indeed, this was observed in our HCQ-receiving cohort, which showed a higher number of associated comorbidities, namely diabetes mellitus, hypertension, and COPD, compared to the control cohort. A meta-analysis of 34 studies conducted by Zhou et al. showed that chronic comorbidities increase the risk of severe course and progression of the disease, with strong correlations with hypertension, diabetes, and CVD [30]. However, there was no difference in rates of CVD presentation between the HCQ-receiving and control groups, which may be due to increased prevalence of CVD in the country [31]. The HCQ-receiving group had significantly less G6PD deficiency, stemming from management guidelines contraindicating HCQ in patients with G6PD deficiency because of increased risk of hemolytic crisis $[32,33]$.

G6PD deficiency has been reported to be disadvantageous against SARS-CoV-2 infections, as the virus prompts a pro-oxidant response while simultaneously suppressing the antioxidant system. Given that a G6PD deficiency results in a compromised antioxidant system, it is postulated that these individuals might suffer more severely from COVID-19 [34]. In addition, ex vivo studies show increased vulnerability of 
Table 4 Risks for developing the primary outcome

\begin{tabular}{lll}
\hline Analysis & Ventilation or death & $P$ value \\
\hline No. of events/no. of patients at risk (\%) & $68 / 1571(4.3 \%)$ & - \\
Hydroxychloroquine & $24 / 440(5.45 \%)$ & - \\
No hydroxychloroquine & $44 / 1131(3.89 \%)$ & - \\
Crude analysis, odds ratio (95\% CI) & $1.43(0.85-2.37)$ & 0.17 \\
Multivariable analysis, odds ratio (95\% CI) & $1.65(0.81-3.32)$ & 0.16 \\
Propensity score analysis, odds ratio (95\% CI) & & 0.24 \\
With matching & $1.75(0.68-4.54)$ & 0.67 \\
With adjustment for propensity score & $0.87(0.47-1.64)$ & \\
\hline
\end{tabular}

Table 5 Risks for developing the primary outcome, in cases who required and did not require supplemental oxygen at baseline

\begin{tabular}{lll}
\hline Analysis & Requiring oxygen at baseline & Not requiring oxygen at baseline \\
\hline No. of events/no. of patients at risk (\%) & $45 / 179(25.14 \%)$ & $23 / 1392(1.65 \%)$ \\
Hydroxychloroquine & $15 / 75(20.00 \%)$ & $9 / 365(2.47 \%)$ \\
No hydroxychloroquine & $30 / 104(28.85 \%)$ & $14 / 1027(1.36 \%)$ \\
Crude analysis, odds ratio (95\% CI) & $1.09(0.38-3.07)$ & $2.79(0.92-8.43)$ \\
\hline
\end{tabular}

Table 6 Risks for developing the primary outcome, in cases who received and did not receive azithromycin

\begin{tabular}{lll}
\hline Analysis & Received azithromycin & Did not received azithromycin \\
\hline No. of events/no. of patients at risk (\%) & $36 / 386(9.3 \%)$ & $32 / 1185(2.7 \%)$ \\
Hydroxychloroquine & $15 / 236(6.36 \%)$ & $9 / 204(4.41 \%)$ \\
No hydroxychloroquine & $21 / 150(14.0 \%)$ & $23 / 981(2.34 \%)$ \\
Crude analysis, odds ratio (95\% CI) & $0.42(0.21-0.84)$ & $1.92(0.87-4.21)$ \\
Multivariable analysis, odds ratio (95\% CI) & $2.71(0.82-8.85)$ & $1.3(0.44-3.75)$ \\
Propensity score analysis, odds ratio (95\% CI) & & $2.12(0.62-7.18)$ \\
With matching & $1.24(0.26-5.8)$ & $1.38(0.59-3.24)$ \\
With adjustment for propensity score & $0.59(0.27-1.31)$ & \\
\hline
\end{tabular}

G6PD-deficient cells to SARS-CoV-2 infection than G6PD normal cells [35]. As a result of their contraindication to receiving HCQ, G6PD-deficient individuals heavily populate the control group, but not the HCQ-receiving group. There is a lack of reports on G6PD and severity of COVID-19 clinical outcomes, and its unknown if G6PD is associated with a more severe disease 
Table 7 Safety outcomes and adverse events

\begin{tabular}{lcl}
\hline Multivariable analysis & Odds ratio $(95 \%$ CI $)$ & $\boldsymbol{P}$ value \\
\hline Hypoglycemia & $10.9(1.72-69.49)$ & 0.011 \\
Diarrhea & $2.8(1.4-5.5)$ & 0.003 \\
QT prolongation & $1.92(0.95-3.9)$ & 0.06 \\
Cardiac arrhythmia & $1.06(0.55-2.05)$ & 0.85 \\
\hline
\end{tabular}

outcome. G6PD is not included within the "high risk" category for COVID-19 in Bahrain, where the study was conducted. If G6PD is associated with a more severe disease then this can potentially be an important confounder in this study. G6PD has been adjusted for through the different statistical analysis used in this study in order to prevent any skew in results. A more thorough analysis is required to accurately assess the association between G6PD and a more severe clinical outcome.

Patients who received HCQ had a higher presentation of symptoms on admission and scored significantly higher on baseline clinical severity scale. Creatinine levels were significantly elevated amongst patients who received HCQ, indicating COVID-19-mediated acute kidney injury [36]. A significantly higher proportion of HCQ receiving patients presented with chest $\mathrm{x}$-ray findings of pneumonia compared to control patients. All these presentations on admission indicate a more severe progression of the disease that is a risk of poor prognosis [28, 36, 37], increasing risk of developing composite outcome and hence indicating
HCQ requirement. This may also explain the higher incidence of composite outcomes seen amongst patients receiving HCQ. It is important to consider that the correlation of higher age and symptomatic status on admission amongst patients receiving HCQ is a result of national guidelines for indication of using HCQ. HCQ was commonly prescribed to these individuals and hence building the difference between the control and the HCQ group.

Interestingly we found no difference in requirement of oxygenation on admission between the HCQ and control groups in our studied sample. This was not expected as requirement of supplemental oxygenation on admission has been associated with increased risk of severe illness [38], and hence expected to be prescribed HCQ. However, this could be interpreted alongside the increased G6PD deficiency amongst the control groups. With a high prevalence of G6PD in Bahrain [39], it may be that many severe COVID-19 admissions, which potentially required oxygen on admission, were contraindicated to receive HCQ.

Almost all factors were insignificant after propensity score matching analysis. However, it is important to note that to conduct matched analysis, the sample size was reduced significantly. The effect of HCQ on the development of the primary outcome remained insignificant using the various ways mentioned to control for confounders.

There was no significant difference in the clinical outcome between HCQ and control groups of patients with mild to moderate disease who did not require oxygen at baseline. Therefore, there is insufficient evidence to

Table 8 Length of stay analysis

\begin{tabular}{lll}
\hline Analysis & Value & $\boldsymbol{P}$ value \\
\hline Overall Mean length of stay for survivors $( \pm \mathrm{SD})$ & $10.0(5.54)$ & - \\
Length of stay in survivor in Hydroxychloroquine group_mean in days $(\mathrm{SD})$ & $11.3(5.65)$ & - \\
Length of stay in survivor in the control group-mean in days $(\mathrm{SD})$ & $9.5(5.41)$ & - \\
Two sample $T$ test: difference & -1.77 & $<0.001$ \\
Multivariable analysis-odds ratio $(95 \% \mathrm{CI})$ & 0.63 & 0.058 \\
& $(95 \% \mathrm{CI}:-0.02$ to 1.3$)$ & \\
\hline
\end{tabular}


suggest benefit of using HCQ to treat patients with low risk of developing severe disease. This finding was consistent across several reports $[40,41]$.

Patients in our study who received HCQ while on oxygen therapy had lower rates of developing the primary outcome, yet this as still non-significant.

Our study showed no clinical benefit from using HCQ in hospitalized patients with COVID-19. Moreover, the effect remained nonsignificant across different subgroups: room air/ oxygen therapy and with and without azithromycin cotreatment.

Our study showed no benefit from combination of azithromycin with HCQ in hospitalized patients with COVID-19. Other studies done in France [10] and Brazil [40] also supported our outcome, and showed no clinical benefit in using the combination of HCQ and azithromycin in the treatment of patients with COVID-19.

It is also important to consider the population sample in this study when interpreting the results. The population used included all COVID-19 cases admitted to Ministry of Health facilities between February and 31 July 2020. Initially during the pandemic, between February and April, all infected cases were admitted to healthcare facilities regardless of symptoms. However only the symptomatic cases or high risk individuals were referred to the acute care hospitals included in our study. Optional home isolation was introduced in May for asymptomatic cases who are younger than 60 years and without comorbidities. Cases who fit home isolation criteria but did not have an appropriate home setup were referred to isolation centers.

Hence, the majority of the population in the study includes patients who required hospitalization due to COVID-19. This limits the study findings to cases requiring hospitalization.

Our study showed that HCQ does not affect the length of hospitalization. The raw analysis showed a significantly higher length of stay compared to patients not receiving HCQ, consistent with several reports $[15,42]$. This is explained by the more severe presentation, higher comorbidities, and risk of lower prognosis leading to the administration of HCQ. Hence, patients who received HCQ would be expected to require a longer stay. Indeed, when these factors were adjusted for in the analysis, the difference was non-significant, which is consistent with other reported data [43]. These findings were also reported in a randomized clinical trial conducted in China on 150 patients with COVID-19. The findings in the trial did not provide evidence to support an increase in the probability of negative conversion of SARS-CoV- 2 conferred by the addition of HCQ to the standard of care in patients admitted to hospital with COVID-19 [41].

With the use of HCQ there was a significant risk of developing adverse effects, specifically hypoglycemia and diarrhea. As a result of their mechanism of action, it is well known that antimalarials cause hypoglycemia. A few studies showed the role of HCQ in patients with diabetes and showed a decreased insulin requirement [44, 45]. As for diarrhea, it is a known adverse effect of HCQ as well [46]. The increased risk of hypoglycemia is alarming, as it would potentially be of a significant risk in patients prone to hypoglycemia or receiving HCQ outside the hospital setting.

The surprising result was the insignificant association between QT interval prolongation and the use of HCQ. It was difficult to find a study that supported our result, as most studies showed frequent prolongation of the QT segment [47]. Our result can be explained by the local protocol used in our hospitals, as daily ECG was done for all patients on HCQ. The local protocol suggests withholding HCQ once QT exceed $470 \mathrm{~ms}$ and can then be restarted once QT has decreased. Moreover patients with a baseline QT greater than $470 \mathrm{~ms}$ or those who are at risk for developing cardiac arrythmia or QT prolongation are seldomly prescribed HCQ.

The findings in our study are supported by findings from multiple clinical trials and observational studies. The RECOVERY trial, which randomized 4716 patients across 176 hospitals in the UK, showed that HCQ had no benefit in decreasing mortality nor invasive ventilation. The findings were consistent across different subgroups including those who received and did not receive oxygen at baseline 
[15]. Another trial conducted in the USA which randomized 479 patients to determine if HCQ improved clinical outcomes at 14 days also supported our results. That multicentered, double-blinded, placebo-controlled study concluded that HCQ did not improve clinical outcomes in patient with COVID-19 respiratory illness. These findings were consistent in all subgroups and for all outcomes evaluated, including an ordinal scale of clinical status, mortality, organ failures, duration of oxygen use, and hospital length of stay [48]. A randomized clinical trial in Brazil was conducted on 667 patients with mild-moderate COVID-19 to measure the effect of HCQ with or without azithromycin on the clinical status at 15 days. The trial concluded that the use of HCQ, alone or with azithromycin, did not improve clinical status at 15 days as compared with standard care [40]. A large observational study was conducted on 1438 hospitalized patients with COVID-19 in New York State to measure the effect of HCQ, with or without azithromycin, on the mortality rates. The study concluded that HCQ, azithromycin, or both, compared with neither treatment, was not significantly associated with differences in in-hospital mortality in patients with COVID-19 [11].

Interpreted along with these prior studies, the results of this study provide additional evidence that HCQ is not beneficial for adults hospitalized with COVID-19.

\section{Strengths}

The study has several strengths. It involved a majority of hospitals which provide acute care for hospitalized COVID-19 cases. Moreover, our study included all hospitals that use HCQ as part of the treatment regimen. The data collection process was done manually and hence all patients files were reviewed carefully and all documented details were collected. The outcomes and adverse events were collected after the medication starting date, and any event occurring within $24 \mathrm{~h}$ of admission or prior to starting the study drug was excluded.

\section{Limitations}

The main limitation of the study is its design, being a retrospective observational study. As a result, the study is limited in its relevancy concerning hospitalized and hence more severe cases of COVID-19 infections. Secondly, given the retrospective design, information that was not documented was not available for analysis, which could lead to potential confounding. This included time from symptom onset, and inflammatory markers. It is also likely that there is still unmeasured residual confounding due to factors not included in the analysis.

\section{CONCLUSION}

Our results showed no significant beneficial effect of using HCQ on the outcome of patients with COVID-19. Moreover, the risk of hypoglycemia due to HCQ would possess a significant risk for out-of-hospital use.

\section{ACKNOWLEDGEMENTS}

We would like to express our gratitude towards our colleagues: Ammar Kheyami, Mujtaba Mal Alla, Abdulla AlMuharraqi, Zeyad Mahmood, Narjis Ali AlSheala, Ola Husain AlHalwachi, Maryam Ghazi Alarayedh, and Amna Mohamed Buheiji who played an essential role in the data collection process related to this paper. Our thanks and appreciation go to them for their hard, dedicated work. Wishing them all the best in the future. We would also like to extend our appreciation to Dr Nitya Kumar and Dr Simone Perna who dedicated time and effort to review and help us improve the manuscript. We also thank The Royal College of Surgeons in Ireland-Medical University of Bahrain for their continuous support of research efforts. We thank all the participants of the study.

Funding. No funding was received to perform this study. The journal's Rapid Service Fee was funded by the authors. 
Authorship. All named authors meet the International Committee of Medical Journal Editors (ICMJE) criteria for authorship for this article, take responsibility for the integrity of the work as a whole, and have given their approval for this version to be published.

Authorship Contributions. Islam AlSayed, Jumana AlArayed, Khadija Alansari, Sara Jaafar Mohammed, Aesha Khalid Sharif and Manaf AlQahtani gathered the data and supervised the data collection team. Abdulkarim Abdulrahman and Abdulla I. AlAwadhi analyzed the data. Abdulkarim Abdulrahman, Manaf AlQahtani, Jumana AlArayed, Sara Jaafar Mohammed wrote the manuscript. Abdulkarim Abdulrahman and Manaf AlQahtani interpreted data and edited the manuscript. All authors reviewed and approved the final version of the manuscript. Manaf Alqahtani is the guarantor of this work.

Disclosures. Abdulkarim Abdulrahman, Islam AlSayed, Marwa AlMadhi, Jumana AlArayed, Sara Jaafar Mohammed, Aesha Khalid Sharif, Khadija Alansari, Abdulla I. AlAwadhi and Manaf AlQahtani have nothing to disclose.

Compliance with Ethics Guidelines. The protocol and manuscript for this study were reviewed and approved by the National COVID19 Research Committee in Bahrain (Approval Code CRT-COVID2020-061). All methods and retrospective analysis of data were approved by the National COVID-19 Research and Ethics Committee, and carried out in accordance with the local guideline and ethical guidelines of the Declaration of Helsinki 1975.

Data Availability. All the data for this study will be made available upon reasonable request to the corresponding author.

Open Access. This article is licensed under a Creative Commons Attribution-NonCommercial 4.0 International License, which permits any non-commercial use, sharing, adaptation, distribution and reproduction in any medium or format, as long as you give appropriate credit to the original author(s) and the source, provide a link to the Creative Commons licence, and indicate if changes were made. The images or other third party material in this article are included in the article's Creative Commons licence, unless indicated otherwise in a credit line to the material. If material is not included in the article's Creative Commons licence and your intended use is not permitted by statutory regulation or exceeds the permitted use, you will need to obtain permission directly from the copyright holder. To view a copy of this licence, visit http://creativecommons.org/licenses/by$\mathrm{nc} / 4.0 /$.

\section{REFERENCES}

1. Cucinotta D, Vanelli M. WHO declares COVID-19 a pandemic. Acta Biomed. 2020;91(1):157-60.

2. World Health Organization. WHO coronavirus disease (COVID-19) dashboard. WHO. 2020. https://covid19.who.int. Accessed 7 Oct 2020.

3. Silva JC, Mariz HA, Rocha LF, et al. Hydroxychloroquine decreases Th17-related cytokines in systemic lupus erythematosus and rheumatoid arthritis patients. Clinics (Sao Paulo). 2013;68(6): 766-71.

4. Liu J, Cao R, Xu M, et al. Hydroxychloroquine, a less toxic derivative of chloroquine, is effective in inhibiting SARS-CoV-2 infection in vitro. Cell Discov. 2020;6:16.

5. Mingo RM, Simmons JA, Shoemaker CJ, et al. Ebola virus and severe acute respiratory syndrome coronavirus display late cell entry kinetics: evidence that transport to NPC1+ endolysosomes is a ratedefining step. J Virol. 2015;89(5):2931-43.

6. Zhou D, Dai SM, Tong Q. COVID-19: a recommendation to examine the effect of hydroxychloroquine in preventing infection and progression. J Antimicrob Chemother. 2020;75(7): 1667-70.

7. Huang C, Wang Y, Li X, et al. Clinical features of patients infected with 2019 novel coronavirus in Wuhan. China Lancet. 2020;395(10223):497-506.

8. Schrezenmeier E, Dörner T. Mechanisms of action of hydroxychloroquine and chloroquine: implications for rheumatology. Nat Rev Rheumatol. 2020;16(3):155-66. 
9. Lenzer J. Covid-19: US gives emergency approval to hydroxychloroquine despite lack of evidence. BMJ. 2020;369:m1335.

10. Gautret P, Lagier JC, Parola P, et al. Hydroxychloroquine and azithromycin as a treatment of COVID-19: results of an open-label non-randomized clinical trial. Int $\mathrm{J}$ Antimicrob Agents. 2020;56(1):105949.

11. Rosenberg ES, Dufort EM, Udo T, et al. Association of treatment with hydroxychloroquine or azithromycin with in-hospital mortality in patients with COVID-19 in New York State. JAMA. 2020;323(24):2493-502.

12. Geleris J, Sun Y, Platt J, et al. Observational study of hydroxychloroquine in hospitalized patients with covid-19. N Engl J Med. 2020;382(25):2411-8.

13. Mehra MR, Desai SS, Ruschitzka F, Patel AN. RETRACTED: Hydroxychloroquine or chloroquine with or without a macrolide for treatment of COVID-19: a multinational registry analysis. Lancet. 2020;1:22.

14. World Health Organization. "Solidarity" clinical trial for COVID-19 treatments. WHO. 2020. https:// www.who.int/emergencies/diseases/novelcoronavirus-2019/global-research-on-novelcoronavirus-2019-ncov/solidarity-clinical-trial-forcovid-19-treatments. Accessed 9 Nov 2020.

15. Horby P, Mafham M, Linsell L, et al. Effect of hydroxychloroquine in hospitalized patients with covid-19. N Engl J Med. 2020;1:2.

16. Bacharier LB, Guilbert TW, Mauger DT, et al. Early administration of azithromycin and prevention of severe lower respiratory tract illnesses in preschool children with a history of such illnesses: a randomized clinical trial. JAMA. 2015;314(19): 2034-44.

17. Andreani J, Le Bideau M, Duflot I, et al. In vitro testing of combined hydroxychloroquine and azithromycin on SARS-CoV-2 shows synergistic effect. Microb Pathog. 2020;145:104228.

18. Marmor MF, Kellner U, Lai TY, Melles RB, Mieler WF, American Academy of Ophthalmology. Recommendations on screening for chloroquine and hydroxychloroquine retinopathy (2016 revision). Ophthalmology. 2016;123(6):1386-94.

19. Costedoat-Chalumeau N, Hulot JS, Amoura Z, et al. Heart conduction disorders related to antimalarials toxicity: an analysis of electrocardiograms in 85 patients treated with hydroxychloroquine for connective tissue diseases. Rheumatology (Oxford). 2007;46(5):808-10.
20. Uzelac I, Iravanian S, Ashikaga $\mathrm{H}$, et al. Fatal arrhythmias: another reason why doctors remain cautious about chloroquine/hydroxychloroquine for treating COVID-19. Heart Rhythm. 2020;17(9): 1445-51.

21. Capel RA, Herring N, Kalla M, et al. Hydroxychloroquine reduces heart rate by modulating the hyperpolarization-activated current $\mathrm{I}_{\mathrm{f}}$ : novel electrophysiological insights and therapeutic potential. Heart Rhythm. 2015;12(10):2186-94.

22. Borba MGS, Val FFA, Sampaio VS, et al. Effect of high vs low doses of chloroquine diphosphate as adjunctive therapy for patients hospitalized with severe acute respiratory syndrome coronavirus 2 (SARS-CoV-2) infection: a randomized clinical trial. JAMA Netw Open. 2020;3(4):e208857.

23. Rismanbaf A, Zarei S. Liver and kidney injuries in COVID-19 and their effects on drug therapy; a letter to editor. Arch Acad Emerg Med. 2020;8(1):e17.

24. Zhang C, Shi L, Wang FS. Liver injury in COVID-19: management and challenges. Lancet Gastroenterol Hepatol. 2020;5(5):428-30.

25. Liu Y, Mao B, Liang S, et al. Association between age and clinical characteristics and outcomes of COVID-19. Eur Respir J. 2020;55(5):1.

26. Chen T, Dai Z, Mo P, et al. Clinical characteristics and outcomes of older patients with coronavirus disease 2019 (COVID-19) in Wuhan, China: a single-centered, retrospective study. J Gerontol A Biol Sci Med Sci. 2020;75(9):1788-95.

27. Chen $\mathrm{T}, \mathrm{Wu} \mathrm{D}$, Chen $\mathrm{H}$, et al. Clinical characteristics of 113 deceased patients with coronavirus disease 2019: retrospective study. BMJ. 2020;368: m1091.

28. Feng Z, Yu Q, Yao S, et al. Early prediction of disease progression in COVID-19 pneumonia patients with chest CT and clinical characteristics. Nat Commun. 2020;11(1):4968.

29. Romero Starke K, Petereit-Haack G, Schubert M, et al. The age-related risk of severe outcomes due to COVID-19 infection: a rapid review, meta-analysis, and meta-regression. Int $\mathrm{J}$ Environ Res Public Health. 2020;17(16):1.

30. Zhou Y, Yang Q, Chi J, et al. Comorbidities and the risk of severe or fatal outcomes associated with coronavirus disease 2019: a systematic review and meta-analysis. Int J Infect Dis. 2020;99:47-56.

31. World Health Organization.. Noncommunicable diseases country profiles 2018. WHO. 2020. https:// www.who.int/nmh/countries/2018/bhr_en. pdf?ua=1. Accessed 2 Nov 2020. 
32. Beauverd Y, Adam Y, Assouline B, Samii K. COVID19 infection and treatment with hydroxychloroquine cause severe haemolysis crisis in a patient with glucose-6-phosphate dehydrogenase deficiency. Eur J Haematol. 2020;105(3):357-9.

33. Nabil A, Uto K, Elshemy MM, et al. Current coronavirus (SARS-CoV-2) epidemiological, diagnostic and therapeutic approaches: an updated review until June 2020. Excli J. 2020;19:992-1016.

34. Buinitskaya Y, Gurinovich R, Wlodaver CG, Kastsiuchenka S. Centrality of G6PD in COVID-19: the biochemical rationale and clinical implications. Front Med (Lausanne). 2020;7:584112.

35. Wu YH, Tseng CP, Cheng ML, Ho HY, Shih SR, Chiu DT. Glucose-6-phosphate dehydrogenase deficiency enhances human coronavirus $229 \mathrm{E}$ infection. J Infect Dis. 2008;197(6):812-6.

36. Cheng Y, Luo R, Wang K, et al. Kidney disease is associated with in-hospital death of patients with COVID-19. Kidney Int. 2020;97(5):829-38.

37. Li J, Chen Z, Nie Y, Ma Y, Guo Q, Dai X. Identification of symptoms prognostic of COVID-19 severity: multivariate data analysis of a case series in Henan Province. J Med Internet Res. 2020;22(6): e19636.

38. Bhargava A, Fukushima EA, Levine M, et al. Predictors for severe COVID-19 infection. Clin Infect Dis. 2020;71(8):1962-8.

39. Al AS. Campaign to control genetic blood diseases in Bahrain. Commun Genet. 2005;8(1):52-5.

40. Cavalcanti AB, Zampieri FG, Rosa RG, et al. Hydroxychloroquine with or without azithromycin in mild-to-moderate Covid-19. N Engl J Med. 2020;383(21):2041-52.
41. Tang W, Cao Z, Han M, et al. Hydroxychloroquine in patients with mainly mild to moderate coronavirus disease 2019: open label, randomised controlled trial. BMJ. 2020;369:m1849.

42. Almazrou SH, Almalki ZS, Alanazi AS, Alqahtani AM, AlGhamd SM. Comparing the impact of hydroxychloroquine based regimens and standard treatment on COVID-19 patient outcomes: a retrospective cohort study. Saudi Pharm J. 2020;28(12): 1877-82.

43. Kalligeros M, Shehadeh F, Atalla E, et al. Hydroxychloroquine use in hospitalised patients with COVID-19: an observational matched cohort study. J Glob Antimicrob Resist. 2020;22:842-4.

44. Rees RG, Smith MJ. Effect of chloroquine on insulin and glucose homeostasis in normal subjects and patients with non-insulin dependent diabetes mellitus. Br Med J (Clin Res Ed). 1987;294(6576):900-1.

45. Blazar BR, Whitley $\mathrm{CB}$, Kitabchi $\mathrm{AE}$, et al. In vivo chloroquine-induced inhibition of insulin degradation in a diabetic patient with severe insulin resistance. Diabetes. 1984;33(12):1133-7.

46. Lofgren SMM, Nicol MR, Bangdiwala AS, et al. Safety of hydroxychloroquine among outpatient clinical trial participants for COVID-19. medRxiv. 2020. 20155531.

47. Agstam S, Yadav A, Praveen Kumar M, Gupta A. Hydroxychloroquine and QTc prolongation in patients with COVID-19: a systematic review and meta-analysis. Indian Pacing Electrophysiol J. 2020. https://doi.org/10.1016/j.ipej.2020.10.002.

48. Self WH, Semler MW, Leither LM, et al. Effect of hydroxychloroquine on clinical status at 14 days in hospitalized patients with COVID-19: a randomized clinical trial. JAMA. 2020;324(21):2165-76. 